\title{
Neuroblastoma intrarenal mimetizando tumor de Wilms
}

\author{
Intrarenal neuroblastoma mimics Wilms' tumor
}

\author{
Maria T. Cartaxo Muniz, ${ }^{1}$ Marcela Araújo, ${ }^{2}$ Leda M. M. Pureza, ${ }^{3}$ Adriana Morais, ${ }^{3}$ Consuelo Antunes, ${ }^{4}$ J osé Romualdo Filho, ${ }^{5}$ Andrezza B. Soares, ${ }^{6}$ \\ Terezinha de J. Marques Salles, ${ }^{7}$ J osenilda C Borges, ${ }^{8}$ Mário H. Magalhães, ${ }^{9}$ Elizabete M. Freitas, ${ }^{10}$ Vera L. L. de Morais ${ }^{11}$
}

\begin{abstract}
Resumo
N o presente trabalho, os autores relatam o caso de uma criança com neuroblastoma intrarenal, que foi, inicialmente, diagnosticado como tumor de Wilms. Pré-escolar, sexo feminino, com um ano e três meses, apresentava uma tumoração endurecida que ocupava o hipocôndrio esquerdo e se estendia até a região do mesogástrio, acompanhada de febre e palidez. 0 ultra-som do abdome total revelou massa intrarenal. A biópsia por agulha fina, em vários pontos de acesso tumoral, revelou um tumor de Wilms. Entretanto, não foi possível naquele momento realizar a imunohistoquímica (IH Q), face à escassez de material. Diante da gravidade da paciente, foi iniciado o protocolo SIO P por quatro semanas. Como não houve resposta clínica, foi indicada uma laparotomia exploradora, com ressecção parcial do tumor, sendo também, nesse momento, realizada punção aspirativa de medula óssea (M 0 ). 0 exame histopatológico revelou neoplasia maligna de pequenas células mal diferenciadas. A IH Q foi negativa para WT-1 e positiva para N B-84, cromogranina esinaptofisina. A biologia molecular revelou amplificação de N -myc. 0 mielograma identificou infiltração medular por pequenas células redondas. 0 neuroblastoma intrarenal é um tumor raro que se assemelha clínica e radiologicamente ao tumor de Wilms. Esse trabalho procura enfatizar a importância do emprego de análises imunohistoquímica e moleculares para o diagnóstico do neuroblastoma intrarenal.
\end{abstract}

Palavras-chave: N euroblastoma intrarenal; Tumor de W ilms; Amplificação N -myc.

\footnotetext{
${ }_{1}^{1}$ Professora de Bioquímica, D outora em Biologia M olecular, Laboratório de Biologia M olecular D epartamento de Biologia, Instituto de Ciências Biológicas/H ospital Universitário O swaldo Cruz, Universidade de Pernambuco, Recife, PE

${ }^{2}$ Bióloga do Centro Infantil Boldrini, Laboratório de Biologia Molecular, Campinas, SP

${ }^{3}$ Oncologista, Centro de O ncologia, H ospital Universitário O swaldo Cruz, Universidade de Pernambuco, Recife, PE

${ }^{4}$ Patologista, Centro de O ncologia do H ospital Universitário O swaldo Cruz, Universidade de Pernambuco, Recife, PE

${ }^{5}$ Patologista, Centro Integrado de Anatomia Patológica, H ospital Universitário O swaldo Cruz, Universidade de Pernambuco, Recife, PE

${ }^{6}$ Bióloga, Laboratório de Biologia M olecular, Departamento de Biologia, Instituto de Ciências Biológicas/H ospital Universitário 0 swaldo Cruz, Universidade de Pernambuco, Recife, PE

${ }^{7} \mathrm{H}$ ematologista, M estre em Biologia M olecular e Celular, Centro de $\mathrm{O}$ ncologia, H ospital Universitário O swaldo Cruz, Universidade de Pernambuco, Recife, PE

${ }^{8}$ Enfermeira, Centro de Oncologia, H ospital Universitário O swaldo Cruz, Universidade de Pernambuco, Recife, PE

${ }^{9}$ Residente em Anatomia Patológica, D epartamento de Patologia, Instituto N acional de Câncer, Rio de Janeiro, RJ

${ }^{10}$ M estre em Genética do Laboratório de Biologia M olecular, D epartamento de Biologia, Instituto de Ciências Biológicas/H ospital Universitário O swaldo Cruz, Universidade de Pernambuco, Recife, PE

${ }_{11}$ Oncologista, Centro de Oncologia, H ospital Universitário O swaldo Cruz, Universidade de Pernambuco, Recife, PE Endereço para correspondência: Prof a D rạ M aria Tereza Cartaxo M uniz. Rua Dr. José M aria 615/203a, Encruzilhada, Recife-PE, CEP: 52041-000 E-mail: tcartaxo@icb.upe.br
} 


\begin{abstract}
This work reports the case history of a child with intrarenal neuroblastoma, initially diagnosed as Wilms' tumor. The patient, a one year and three months old girl, presented a hard abdominal mass on the left flank that extended to the mesogastric region, plus fever and paleness. The ultrasound of the entire abdomen revealed an intrarenal mass. Biopsy with fine needle in many points of the tumor revealed W ilms' tumor. The scarcety of the material, however, made immunohistoquemistry impossible at that moment. Because of the child's severe condition the SIOP protocol was started. As no clinical response was observed, an exploratory laparatomy was indicated with partial resection of the tumor and bone marrow aspiration (M 0 ). The histopathologic study reveal ed a malignant neoplasia of small cells, poorly differentiated. IH Q was negative for WT-1 and positive for N B-84, synaptofisin, cromogranine. $\mathrm{N}$-myc amplification was observed by molecular biology. The bone marrow aspiration identified matastatic small round cells infiltration. Intrarenal neuroblastoma is a rare entity that clinically and radiographically resembles W ilms' tumor. The objective of this case report is to show the importance of immunohistochemical and molecular analysis in the diagnosis of intrarenal neuroblastoma.
\end{abstract}

Key words: Intrarenal neuroblastoma; Wilm's tumor; $\mathrm{N}$-myc amplification.

\section{INTRODUÇÃO}

Tumor de Wilms é a neoplasia intrarenal sólida mais comum da infância, com uma incidência de um em cada 100.000 casos. ${ }^{1}$ Se por um lado, a princípio, qualquer massa encontrada no rim de uma criança sugere tumor de Wilms até que se demonstre 0 contrário. ${ }^{2}$ Por outro lado, neuroblastoma é o tumor sólido extracranial mais comum da infância e pode se originar em qual quer tecido do sistema nervoso simpático, da cabeça até a pelvis, com predominância na medula adrenal. 0 neuroblastoma abdominal comumenteestende-se aos linfonodos etecidos adjacentes, mas pode haver infiltração do parênquima renal. ${ }^{3}$ Acredita-se que o neuroblastoma intrarenal pode ser originário de restos da adrenal dentro do rim ou de gânglios simpáticos intrarenais. ${ }^{4} \mathrm{C}$ omo 0 neuroblastoma intrarenal ocorre muito raramente, esse tipo de enfermidade, ainda, não foi bem caracterizado. ${ }^{5}$

\section{RELATO DO CASO}

U ma criança de um ano e três meses de idade, sexo feminino, apresentava uma massa abdominal no lado esquerdo, associado a febre e palidez. 0 ultra-som do abdome total (figura 1) mostrava uma massa tumoral sem plano de clivagem com o rim esquerdo medindo $14,1 \mathrm{x}$ $11,5 \times 11,2 \mathrm{~cm}$. A tomografia computadorizada do abdome total (figura 2) revelou um processo expansivo sólido, acometendo o rim esquerdo, ultrapassando a linha média com grandes áreas de necrose e envolvendo as estruturas vasculares retroperitoniais, chegando inclusive a relacionar-se com a veia renal direita. 0 hemograma

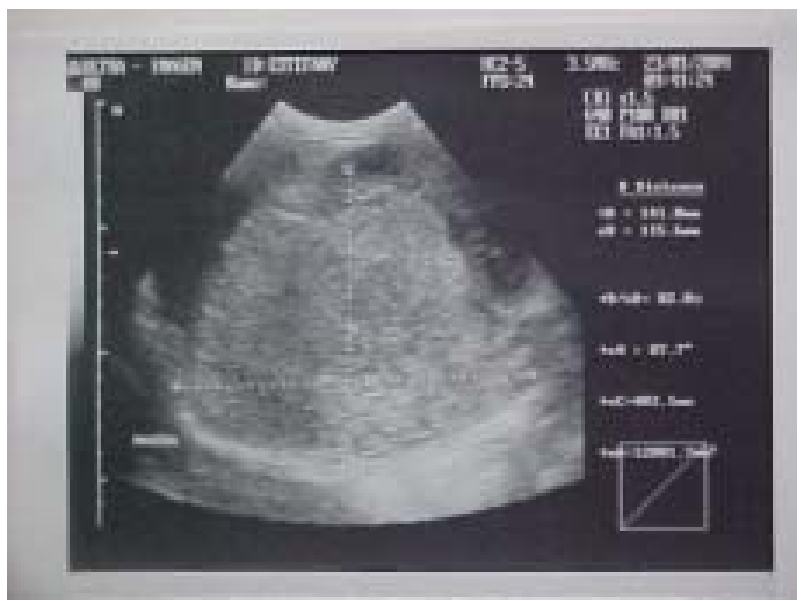

Figura 1. Ultra-sonografia de abdome total mostrando presença de volumosa massa sólida heterogênea, localizada no hemi abdome esquerdo, medindo $14,1 \times 11,5 \times 11,2 \mathrm{~cm}$, apresentando relação anatômica lateralmente com 0 rim esquerdo.

revelou $\mathrm{H}$ ematimetria $2.300 .000 / \mathrm{mm}^{3}$, hematócrito $16 \%$ e hemoglobina 5,2g/dL. A desidrogenase láctica $(D H L)$ foi de $5010 \mathrm{UI} / \mathrm{L}$. O s ácidos vanilmandélico (VM A) e homovanílico (HVA) foram solicitados e não determinados. A paciente evoluiu com agravamento do quadro eanasarca. A primeira biópsia realizada por agulha fina revelou tumor de Wilms.

Pela gravidade do estado geral da paciente, a terapia foi iniciada com o protocolo SIOP (SIOP, 2001) para tumor de W ilms: primeira semana, vincristina e actinomicina $D$; segunda semana, vincristina; terceira semana, vincristina e actinomicina $D$ e quarta semana, vincristina. $\mathrm{N}$ ão houve resposta ao tratamento. Foi então solicitada uma laparotomia exploradora com ressecção 
parcial do tumor e punção aspirativa de M 0 . 0 exame anatomopatológico revelou neoplasia maligna de pequenas células mal diferenciadas, a IH Q foi negativa para W T-1 e positivo a para NB-84, cromogranina e sinaptofisina. A biologia molecular revelou amplificação de $\mathrm{N}$-myc (figura 3), fechando assim o diagnóstico deneuroblastoma. Foi, então, iniciado o protocolo VECI (vincristina d1, ifosfamida - d1 e d2; carboplatina - d3 em infusão de 24 horas; e Teniposide - d4). A paciente continuou apresentando progressão da doença e evoluiu para o óbito.

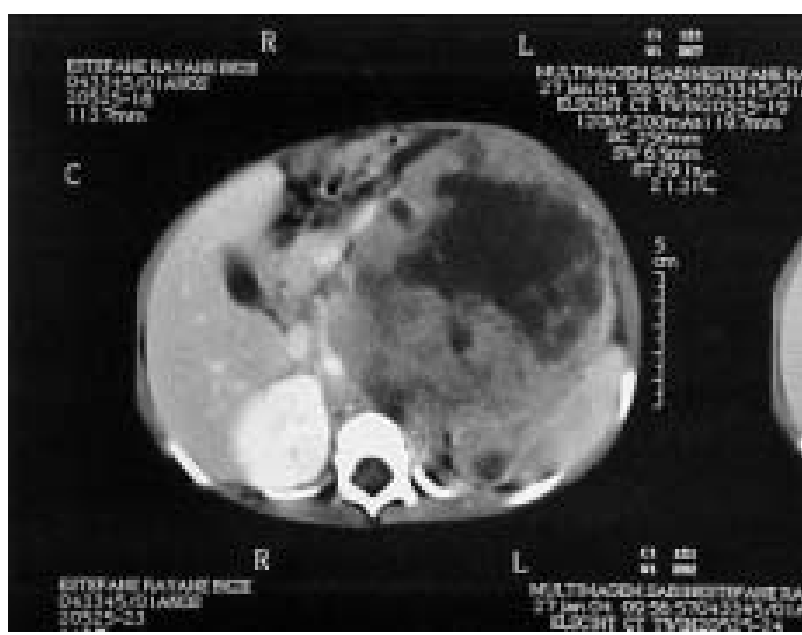

Figura 2. Tomografia computadorizada do abdome total com contraste mostrando a presença de volumoso processo expansivo sólido com extensas áreas de necrose comprometendo o rim esquerdo, cujo epicentro é visto na loja renal esquerda e medindo $13,2 \times 13,0$ $\mathrm{X} 10,5 \mathrm{~cm}$.

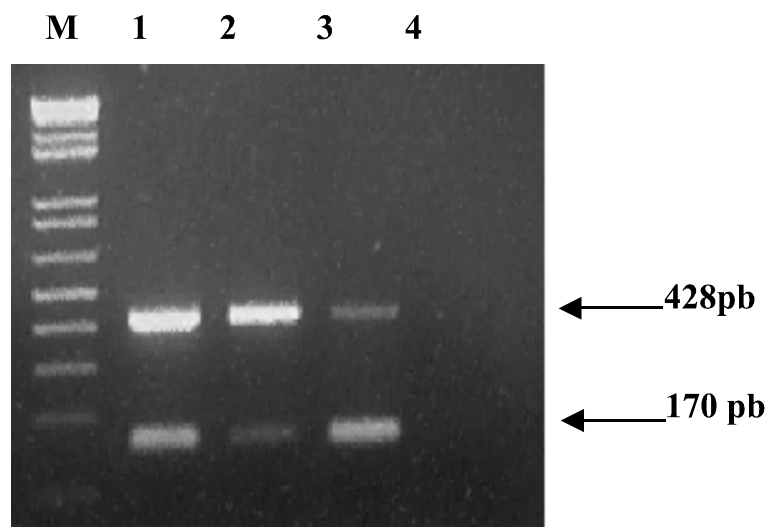

Figura 3. Gel $1 \%$ com brometo de etídio para produtos amplificados de PCR. As bandas superiores representam produtos amplificados do gene $\mathrm{N}$-myc $(428 \mathrm{pb})$. As bandas inferiores correspondem ao produto de amplificação do gene CF (170 pb). As linhas 1 a 4 correspondem ao paciente $(P)$, controle positivo $(C+)$, controle negativo (C-) e branco (W), respectivamente. $M$, marcador molecular $1 \mathrm{~Kb}$ Plus DNA Ladder.

\section{DISCUSSÃO}

Relatamos o caso de uma menina de um ano e três meses com uma massa intrarenal que clínica e radiologicamente simulava um tumor de W ilms.

N euroblastoma é o tumor sólido extra-craniano mais comum, perfazendo de 8 a 10\% de todos os cânceres da infância. ${ }^{6}$ Acomete cerca de $50 \%$ das malignidades neonatais e pode ocorrer em qualquer região ao longo do sistema neuroectodermal simpático, da cabeça a pélvis, embora $50-70 \%$ dos casos ocorram na medula adrenal. ${ }^{5} \mathrm{H}$ istologicamente, o neuroblastoma pertence ao grupo de tumores de células pequenas, redondas e azuis da infância, que incluem sarcoma de Ewing, rabdomiossarcoma embrionário e linfomas. Os neuroblastomas bem diferenciados exibem uma formação em roseta e neurofibrilas evidentes. Contudo, tumores pobremente diferenciados são mais difíceis de distinguir de outros tumores anaplásicos à miscroscópia óptica e requerem uma avaliação mais específica, como a presença da amplificação $\mathrm{N}$-myc. ${ }^{7}$ Apesar de outros tumores poderem apresentar amplificação do gene $\mathrm{N}$ myc, a hipótese clínica e o conjunto de exames laboratoriais podem definir o diagnóstico.

A literatura mostra poucas descrições de neuroblastoma intrarenal. Serrano et al. descreveram dois casos de neuroblastoma intrarenal primário diagnosticado por aspiração com agulha fina (FNA) , guiado por tomografia computadorizada. ${ }^{4} \mathrm{M}$ CQ uaid e 0 'M eara estudaram a amplificação de $\mathrm{N}$-myc em 29 pacientes, dos quais, 14 casos eram de neuroblatoma, 9 de tumor de W ilms e em 6 outros tumores. Esses achados indicaram que a ativação do $\mathrm{N}$-myc, pela amplificação, confere propriedades agressivas em uma variedade de tumores embrionários. ${ }^{8} \mathrm{~K}$ essler et al. descreveram 5 crianças com massa abdominal inicialmente diagnosticadas como tumor de W ilms, mas o quadro de hipertensão em todos os casos, e as calcificações típicas em dois deles levantaram a suspeita de neuroblastoma. Foi observada amplificação de $\mathrm{N}$ myc em todos os casos e dois desses responderam ao tratamento, um deles, inclusive apresentando alto grau de amplificação de $\mathrm{N}$-myc, o qual foi submetido a um transplante autólogo, três pacientes foram a óbito. ${ }^{5}$

$\mathrm{N}$ o presente relato, o diagnóstico de neuroblastoma foi confirmado pelo conjunto de exames, entre eles 0 exame anatomopatológico, a punção aspirativa de M 0 , a análise imunohistoquímica e biologia molecular, em face dos métodos de imagem serem insuficientes para identificação dessa massa abdominal. Esse caso representa mais um relato de neuroblastoma intrarenal, uma forma rara de manifestação dessa neoplasia. 


\section{REFERÊNCIAS}

1. Young L L, M iller RW. Incidence of malignant tumorsin US children. J Pediatr. 1975;86:254-8.

2. Shende A, W ind SE, Lankowsky P. Intrarenal neuroblastoma mimicking W ilms tumor. N Y StateJ M ed. 1979;79:93.

3. Panuel $M$, Bourliere-N ajean B, G entet JC, Scheiner $C$, D elarue A, Faure F, et al. Aggressive neuroblastoma with initial pulmonary metastases and kidney involvement simulating W ilms' tumor. EurJ Radiol. 1992;14:201-3.

4. Serrano R, Rodriguez-Peralto J $L, D$ eO rbe G G, M elero C , deAugustin A. Intrarenal neuroblastoma diagnosed by fine- needleaspiration: report of two cases. D iagn Cytopathol. 2002;27(5):294-7.

5. Kessler OJ, Siegel JF, Brock WA. Intrarenal neuroblastoma masquerading asW ilms tumor. U rology. 1998;51(2):313-6.

6. Pizzo PA, Poplack D G. Principles and pratice of pediatric oncology. 4th ed. Philadelphia: Lippincott W illiams \& Wilkins; 2002.

7. $N$ isen $P D$, Rich M A, Gloster E, Valderrama E, Saric $O$, Shende $\mathrm{A}$, et al. $\mathrm{N}$-myc oncogene expression in histopathologically unrelated bilateral pediatric renal tumors. C ancer. 1988;61(9):1821-6.

8. $M \subset Q$ uaid $S, O$ 'M earaA. $N$-myc oncogeneamplification in paediatric tumors. Ir J M ed Sci. 1990;159(6):172-4. 